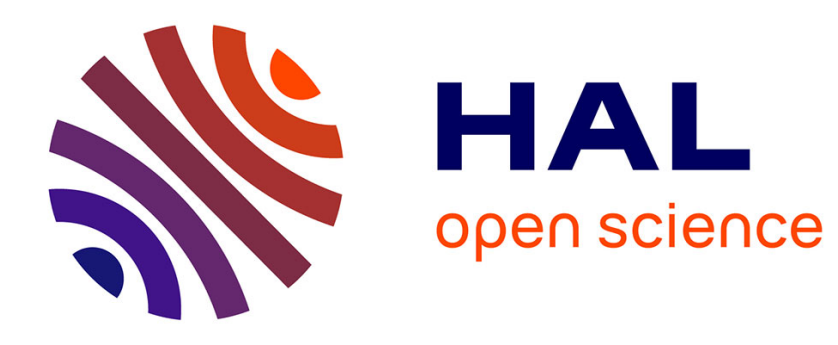

\title{
Penser le passé, parler du passé: l'identité afrikaner chez Mark Behr et Antjie Krog
}

Myriam Houssay-Holzschuch

\section{To cite this version:}

Myriam Houssay-Holzschuch. Penser le passé, parler du passé : l'identité afrikaner chez Mark Behr et Antjie Krog. Travaux de l'Institut de Géographie de Reims, 1999, 25 (99/1000), pp.157-168. hal00186059

\section{HAL Id: hal-00186059 \\ https://hal.science/hal-00186059}

Submitted on 7 Nov 2007

HAL is a multi-disciplinary open access archive for the deposit and dissemination of scientific research documents, whether they are published or not. The documents may come from teaching and research institutions in France or abroad, or from public or private research centers.
L'archive ouverte pluridisciplinaire HAL, est destinée au dépôt et à la diffusion de documents scientifiques de niveau recherche, publiés ou non, émanant des établissements d'enseignement et de recherche français ou étrangers, des laboratoires publics ou privés. 


\title{
Penser le passé, parler du passé :
}

\section{l'identité afrikaner chez Mark Behr et Antjie Krog}

\author{
Myriam HOUSSAY-HOLZSCHUCH \\ Géographe \\ Université de Reims Champagne-Ardenne
}

\begin{abstract}
"A cause de la musique pop, vous pouvez devenir un drogué. Avant que Lucifer soit chassé du ciel, il était l'ange de la musique et c'est pour ça que c'est logique que les Communistes utilisent la musique pop pour s'emparer de la République. Les Beatles ont même dit qu'ils étaient plus importants que Jésus. Les Beatles et Cat Stevens sont vraiment les instruments de Lucifer et de l'Antéchrist, mais l'humanité est trop stupide pour lire ce qui est écrit en lettres de feu"

"A chaque victime ayant un nom afrikaner sur les lèvres"
\end{abstract}

L'un des problèmes auxquels la nouvelle Afrique du Sud est confrontée depuis les débuts de la transition démocratique est de parvenir à construire une unité nationale, intégrant chaque partie de la population tout en respectant une diversité culturelle parfois hautement revendiquée, que ce soit par exemple par les Zoulous ou par les Afrikaners. Ce souci a été exprimé tout au long de la transition et pendant les premières années du gouvernement Mandela par différents partis politiques et groupes de pression : c'était un facteur important dans les négociations multipartites des années 1990-1994 et il a été aussi l'une des données de base de la politique de réconciliation nationale prônée par Nelson Mandela, Desmond Tutu et d'autres.

Cette réconciliation nationale doit, dit-on, se faire entre Noirs d'un côté et Blancs de l'autre, entre opprimés et oppresseurs, entre pauvres et riches. Mais la situation est évidemment plus complexe : dans ce face-à-face entre Noirs et Blancs, les Afrikaners entre autres jouent un rôle particulier. Même si la ségrégation et bien des lois l'imposant étaient en place avant leur accession au pouvoir en 1948, ils sont les principaux responsables de la mise en place de l'apartheid (Houssay-Holzschuch, 1996). Leur identité est partiellement fondée sur une conception exclusive de la "race" et du peuple. Ce sont eux ou, à tout le moins, leur élite qui possédaient le pouvoir et l'ont cédé à un gouvernement élu au suffrage universel : " they have negotiated themselves out of power", suivant l'expression de Adam, Slabbert et Moodley (1997). Ils doivent aujourd'hui trouver leur place dans la nouvelle nation sud-africaine, que certains n'ont pas encore acceptée et dans laquelle ils n'ont plus un rôle prédominant. C'est une entreprise longue et difficile, qui soulève des problèmes fondamentaux pour la jeune démocratie : celui de la diversité culturelle dans un état unitaire bien sûr, mais aussi ceux du langage et de la relecture du passé d'apartheid.

Dans cette entreprise, écrivains et poètes de langue afrikaans ne sont pas restés inactifs, suivant en cela une tradition bien établie, des chantres du nationalisme afrikaner aux écrivains plus critiques et rebelles des années 1960. En particulier, l'identité afrikaner passée et présente est le thème central de deux ouvrages récents : The Smell of Apples de Mark Behr (1993) et Country of my Skull d'Antjie Krog (1998). Les deux auteurs publient en anglais ${ }^{3}$, ce qui est déjà une déclaration d'intention, et cherchent à comprendre comment leur peuple a pu commettre de telles choses. La forme adoptée est très différente : Behr

${ }^{1}$ BEHR (1995). The Smell of Apples. London, Abacus, p. 67. Les citations anglaises ont été traduites par mes soins.

${ }^{2}$ KROG (1998). Country of my Skull. Johannesburg, Random House, dédicace.

${ }^{3}$ L'ouvrage de Behr a aussi été publié en afrikaans. 
écrit pendant la période de négociation, avant les élections d'avril 1994. Il choisit le roman et un narrateur enfant évoquant sa vie quotidienne au sein d'une bonne famille afrikaner de la ville du Cap, en 1974. C'est son premier livre. Krog, poétesse reconnue en langue afrikaans, a suivi comme journaliste pour la radio les auditions de la Commission Vérité et Réconciliation (Truth and Reconciliation Commission), chargée de faire la lumière sur les crimes commis durant la période d'apartheid (Aji et Houssay-Holzschuch, 1997) et son livre narre cette expérience. Mais les mêmes thèmes reviennent dans chacun des deux ouvrages : quelle est cette culture, cette identité afrikaner qui a produit l'apartheid ? Comment les pères, les cousins, avec qui l'on a vécu pendant des années, en les aimant et en les comprenant, ont pu à ce point incarner le Mal ? Comment être afrikaner aujourd'hui, assumer cette histoire tout en la condamnant et faire partie de la nouvelle Afrique du Sud ?

\section{L'ordre d'un monde}

La constitution d'une identité afrikaner n'a pas été chose facile et rapide. Bien au contraire, chaque famille afrikaner, descendant des premiers colons néerlandais, allemands et français, était attachée à son indépendance, politique, économique et religieuse. Cette volonté d'autonomie est soulignée encore aujourd'hui par certains intellectuels afrikaners (Giliomee, 1996). Pour établir une unité, construire une nation afrikaner, il a fallu qu'un groupe d'intellectuels entreprenne une série d'actions concertées et volontaristes tant sur le plan culturel que linguistique, économique ou politique (Houssay-Holzschuch, 1996 et 1999). Ce regroupement s'est fait autour d'un certain nombre de thèmes fédérateurs comprenant entre autres : une référence permanente au religieux, permettant d'établir un parallèle entre l'Exode du peuple élu vers la Terre promise et les tribulations des Afrikaners en Afrique australe ; une vision téléologique de l'histoire ; une conception de l'Autre, Anglais ou Noir, marquée par le culte de la différence et des spécificités culturelles. L'arrivée au pouvoir du Parti national en 1948, élu sur un programme prônant la systématisation de la ségrégation raciale (apartheid), a modifié l'espace sud-africain à toutes les échelles : il devait correspondre à une certaine idéologie et a permis à une certaine vision de l'Afrikanerdom, de la nation et de l'identité afrikaner, d'atteindre son apogée.

Le roman de Mark Behr s'attache à décrire les caractéristiques de cette identité afrikaner et la façon dont elle s'exprime dans la vie quotidienne et familiale. Pour atteindre son but, il choisit de situer l'action au début des années 1970, soit au moment où l'on peut croire que l'apartheid est un succès : la résistance noire des années 1950 et 1960 a été réprimée dans le sang, la loi et l'ordre semblent régner, la politique du grand apartheid s'attache à la constitution des homelands, Oom (oncle) John Vorster et Oom P. W. Botha veillent sur le pays. Par petites touches, Behr précise l'époque en faisant référence au contexte international : les États-Unis finissent de s'enliser au Vietnam, le premier choc pétrolier a déjà eu lieu ainsi que le coup d'état au Chili : la famille Erasmus est décrite en 1974, soit à la veille des émeutes de 1976, de la renaissance d'une résistance noire forte, armée et agissant sur le territoire sud-africain. Les signes avant-coureurs sont déjà là, pour qui ne se contente pas de la propagande officielle : la grande grève des dockers de Durban en 1972 est évoquée (p. 107).

Cette exploration de l'identité afrikaner se fait presque uniquement au sein du cercle familial. Le narrateur, Marnus Erasmus, a dix ans. Il fréquente l'une des écoles destinées à l'élite afrikaner, est chef de patrouille au sein du mouvement de jeunesse contrôlé par le Parti national, les Voortrekkers. Il joue au rugby et au cricket, ressemble à son père qu'il souhaite combler : bref, c'est un jeune garçon afrikaner de bonne famille. Johann Erasmus, $\mathrm{Dad}$, est le personnage central du roman : cultivé, beau et soigné, parlant l'anglais aussi bien que l'afrikaans et ayant fait ses études à West Point, il est aussi le plus jeune major général de l'armée sud-africaine. Il fait partie de la société secrète afrikaner et élitiste du Broederbond, a combattu les "terroristes" en Rhodésie et travaille pour les Renseignements. C'est à ce titre que sa famille accueille des militaires français, israéliens et chiliens, sous de faux noms, pendant leurs séjours en Afrique du Sud. Leonore, la mère, est une ancienne cantatrice. Toujours belle, blonde et élégante, elle s'occupe de la maison 
au bord de la mer, de Marnus et de sa sœur aînée Ilse, de la bonne et du jardinier, tous deux métis (Coloured).

Les personnages de Behr ne sont pas de typiques Afrikaners : ils appartiennent à l'élite et ne font pas partie de ces "pauvres Blancs" en faveur desquels le gouvernement d'apartheid va œuvrer. Ils ne sont plus liés à un terroir rural du nord du pays, contrairement à ceux qu'évoque Antjie Krog, et les seuls paysages fondateurs de l'ouvrage sont le Tanganyika, que la famille de Johann a dû quitter lors des luttes pour l'indépendance, et False Bay, au sud de Cape Town. Pourtant, c'est parce que nous sommes au cœur du système d'apartheid que son analyse va être possible. Le choix du narrateur, un enfant de dix ans, va permettre de mettre en lumière le langage du pouvoir. Marnus reprend mot pour mot la propagande gouvernementale exactement comme il répète les lois édictées par ses parents : le langage de l'autorité est partout le même, il a la même valeur d'impératif moral ${ }^{4}$. Constamment, il va reprendre des formules toutes faites :

\footnotetext{
"Au début, je ne voulais pas laisser [Frikkie copier sur moi] parce que Maman dit que copier le devoir de quelqu'un d'autre, c'est la même chose que de voler avec ses yeux. Qu'on vole quelque chose avec ses mains ou le devoir de quelqu'un d'autre avec ses yeux, le péché est aussi grand et aussi grave" (Behr, op. cit., p. 5).

"Mais maintenant les Noirs essaient de faire à la République exactement ce qu'ils ont fait au Tanganyika. Ils essaient de s'emparer de tout ce que nous avons construit au cours des années, juste pour le détruire comme ils détruisent tout ce qu'ils touchent. De toutes les nations du monde, celles qui ont de la peau noire sur les fesses sont celles qui ont les plus petits cerveaux. Même si vous arrivez à sortir le Noir de la brousse, vous n'arriverez jamais à sortir la brousse du Noir. [...].

Les Bantous sont encore plus bêtes que les métis ["Coloureds "]. Heureusement, les métis ont encore un peu du sang des marins dans leurs veines. Mais maintenant il y en a si peu que les métis sont presque tous des ivrognes qui boivent toute leur paie le week-end. Très souvent, ce sont des criminels qui n'iront jamais au Paradis. Saint Pierre, qui garde la porte de l'éternité, tombera dans les pommes quand il sentira leur haleine.

Mais Doreen [la bonne] est une bonne fille et elle pourrait bien aller au paradis. Au paradis, elle vivra avec les autres métis chrétiens dans des petites maisons et le Seigneur la récompensera de ne s'être jamais saoulée comme les autres. Et de n'avoir jamais piqué le sucre de Maman [...]" (Ibid., pp. 38-39)
}

L'ordre familial, l'ordre gouvernemental et l'ordre divin sont donc organisés de la même manière, protégés par les mêmes lois, les mêmes discours et les mêmes autorités : ce sont les mêmes. Ce sont aussi des ordres patriarcaux, où les femmes s'effacent et où Marnus est surnommé " le petit taureau ". Cette importance de la virilité dans la culture afrikaner est aussi exprimée par Krog, cette fois de façon négative et consciemment associée à la violence et à l'ancien régime d'apartheid. Elle décrit ici l'arrivée des Afrikaners les plus réactionnaires au Parlement et l'arsenal de leur machisme :

“Écrasées sur leurs amortisseurs, trois Sierra blanches et abîmées passent en rugissant à travers les portails de fer forgé menant au Parlement. De lourds avant-bras, pareils à des jambons, dépassent par les fenêtres ouvertes - ils klaxonnent, ils agitent les anciens drapeaux de l'État libre et du Transvaal. Des poings velus sont brandis " (Krog, op. cit., p. $1)$.

Cercle familial, société patriarcale très hiérarchisée, importance de la religion dans la vie quotidienne et politique, adhésion totale et immédiate à tout discours d'autorité teinté de morale et de calvinisme : telles sont selon Behr les caractéristiques de l'identité afrikaner sous l'apartheid. En même temps, cette société est convaincue de faire le bien, sur le plan international comme vis-à-vis des autres populations sud-africaines. Double langage et hypocrisie ou juxtaposition permanente du Bien et du Mal ?

\footnotetext{
${ }^{4}$ Cette identité entre les deux principaux discours d'autorité est soulignée par le fait que les gouvernants sont appelés "Oncle” (Oom en afrikaans).
} 


\section{Une géographie quotidienne du Mal}

Les rapports entre culture afrikaner et origine du mal - l'apartheid - sont au centre de l'exploration identitaire de Behr et de Krog. Là encore, les solutions qu'ils choisissent sont différentes. Behr construit le développement de son intrigue sur la découverte que Marnus jeune fait de la "part d'ombre" de ses parents. Krog utilise l'histoire récente et les témoignages des victimes comme des bourreaux devant la Commission Vérité et Réconciliation. Mais que la découverte du mal se fasse dans un contexte romanesque ou dans celui du journalisme d'actualité de la nouvelle Afrique du Sud, elle est doublement choquante et traumatisante. D'abord, par l'ampleur du mal commis et par son atrocité. Ensuite, parce que les bourreaux sont si proches, culturellement et affectivement, des narrateurs qui dénoncent le mal qu'ils commettent.

Pour Marnus, le mal s'inscrit au cœur même de la famille qu'il décrit et qu'il aime, et le bourreau est son père si admirable. Tout se passe au cours d'une soirée familiale, détendue et sereine, la dernière que le général chilien qu'ils reçoivent depuis quelques jours passe avec eux. Dad y montre des diapositives du Tanganyika et de Rhodésie, ces dernières prises lors de la guerre de libération où il combattait contre les "terroristes" aux côtés des forces rhodésiennes. Les premiers clichés le montrent en tenue d'officier, donnant des ordres. Les suivants sont aussi projetés devant le général, Leonore, Ilse, Marnus et son ami Frikkie :

\footnotetext{
"La diapositive montre quatre terroristes nus, debout dans une clairière. Leurs mains sont attachées au-dessus de leur tête et un soldat tient une baïonnette contre la poitrine de l'un d'entre eux. On peut voir le blanc de ses yeux dans son visage noir. Il est peut-être en train de pleurer car son visage est crispé comme s'il hurlait. [...] Maintenant, les quatre terroristes sont entassés et on peut voir qu'ils ont été abattus. Leurs corps sont couverts de sang. Celui qui était devant sur la diapositive précédente a les jambes écartées vers l'appareil photo et son machin noir est allongé presque jusque par terre " (Behr, op. cit., p. 171).
}

D'autres clichés de torture sont suivis par des vues touristiques de Luanda, au Mozambique : la séance se termine. Mais plus tard dans la soirée, Marnus est témoin du viol de son ami Frikkie par son père - manifestement une habitude ; il voit aussi sa mère passer la nuit dans les bras du général chilien. Le mal est présent et agissant au sein même de la famille, le cœur du système politique afrikaner.

Pour Krog, la description de la Commission Vérité et Réconciliation suit la chronologie des événements : mise au point de l'appareil législatif nécessaire, constitution de la Commission, témoignages des victimes, arrivée et témoignages des bourreaux. Les victimes avaient déjà montré l'ampleur du mal en le narrant en mots simples :

\footnotetext{
"C'est à l'intérieur de moi... cela empêche ma langue. Cela ne peut pas... être partagé. Cela détruit... les mots. Avant qu'on fasse exploser son corps, ils lui ont coupé la main pour qu'on ne puisse pas prendre ses empreintes... Comment puis-je dire ? Cette terrible... Je voudrais qu'on me rende sa main " (Krog, op. cit., p. 27).
}

Quand les bourreaux arrivent, Krog les reconnait. Dirk Coetzee, commandant du plus cruel des escadrons de la mort, Vlakplaas, cultivé et ambigu est à l'image du père de Marnus, devant rendre compte de ses actes vingt ans plus tard. Quant aux exécutants, leur accent, leur manière d'appeler, comme Dad, leur fils " mon petit taureau ", leur attitude toute entière lui est familière. Pour pouvoir les interviewer, elle fait appel au langage, aux attitudes qui lui étaient naturels pendant son enfance : "Ils me sont aussi familiers que mes frères, mes cousins et mes camarades d'école. Entre nous, toute distance est effacée. Peut-être n'y a-t-il jamais eu de distance entre nous, à l'exception de celle que j'ai péniblement bâtie en moi-même au cours des ans?" (Krog, op. cit., p. 96).

Devant cette proximité du mal, la réaction de Marnus et celle de Krog diffèrent. Malgré sa prise de conscience, le mal dont Marnus prend conscience est trop proche pour qu'il puisse le condamner. Les tortures commises par les troupes de son père sont justifiées par la propagande gouvernementale. Le viol de Frikkie instaure simultanément méfiance et compréhension dans sa relation avec ses parents. Il cherche à le faire parler sur ce qui lui est arrivé, mais en vain. Et conclut: "Et je sais que si [Frikkie] veut dire 
quelque chose à quelqu'un, quelque chose que personne d'autre ne doit savoir, alors il le dit à moi et seulement à moi. S'il n'a même pas voulu me dire ce qui s'est passé avec Papa, il ne dira jamais rien à personne. Et c'est bien comme ça" (Behr, op. cit., p. 199).

Marnus protège le bourreau. Plus encore, son compte-rendu de l'identité afrikaner est fait à deux voix : la narration au présent de Marnus enfant est entrecoupée de passages postérieurs, où l'officier Marnus mène une guerre sale et clandestine dans le sud de l'Angola, dans la première moitié des années 1980. Poussière, désespoir, sentiment d'être abandonné et ignoré de l'arrière : la radio des troupes retransmettra même une interview de Dad, alors à l'état-major, affirmant qu'il n'y a aucun soldat sud-africain en Angola. Son fils l'écoute, sous les obus cubains. La guerre est là une seconde prise de conscience de la vacuité du discours officiel, du mal que l'on commet. Elle sera pourtant vaine : l'officier Marnus meurt sur le champ de bataille.

L'ouvrage de Krog tout entier est une réaction au mal dévoilé. Son travail de journaliste cherche à le faire connaître et reconnaître : elle-même doit d'abord l'accepter et l'assumer, puis tenter que les Afrikaners, les Sud-Africains, voire le reste de l'humanité en soit conscient.

\section{Groupe, individu, nation}

Chacune de ces œuvres témoigne de la façon dont la transition démocratique est vécue au sein de la population afrikaner. L'une et l'autre sont représentatives des phases politiques pendant lesquelles elles ont été écrites. Mark Behr écrit en 1993: les négociations d'avant les élections sont encore en cours. L'année précédente, la population blanche a répondu par l'affirmative à un référendum sur la poursuite des réformes et des négociations. Elle est encore persuadée d'agir - et d'avoir agi - pour le bien de l'ensemble de la nation sud-africaine. C'est l'époque où F. W. de Klerk, en s'excusant pour les crimes commis sous l'apartheid, le décrit comme une politique généreuse, pleine de bonnes intentions, mais qui aurait mal tourné. The Smell of Apples contredit cette interprétation, en montrant le mal intrinsèque au mode de vie et à la pensée des maîtres de l'apartheid. En même temps, l'œuvre est d'abord littéraire : la transition permet d'adopter un langage qui n'est plus uniquement politique, qu'il se cache ou non sous une forme littéraire pour échapper à la censure.

Krog, poétesse, choisit une forme qui lui est étrangère : le journalisme. Comme Behr mêlait romanesque et éléments autobiographiques, elle utilise à la fois le reportage, l'introspection et des passages de fiction où elle reconstruit la / une / sa vérité. La fiction est pour elle le moyen de parvenir à une vérité plus grande, voire le moyen de l'exprimer lorsqu'elle touche à l'indicible. Elle suit l'actualité de la Commission Vérité et Réconciliation, les atermoiements des politiciens venus témoigner et la lente révélation de la vérité : dans un langage poétique, les principaux événements de la vie politique sudafricaine depuis le massacre de Sharpeville en 1960 défilent. Assassinat de Steve Biko, affaire Winnie Mandela, attentats contre des activistes de l'ANC en dehors des frontières nationales, sont évoqués par les victimes et leurs proches comme par les bourreaux.

Il s'agit toujours, pour Behr comme pour Krog, de travailler sur le langage. Le problème est bien loin d'être neutre : c'est autour de la reconnaissance de leur langue, l'afrikaans, que l'identité afrikaner s'est structurée. Aujourd'hui, la place de la culture afrikaner au sein de l'État sud-africain unitaire et multiculturel est évoquée par le biais du langage. Il s'agit là d'un problème fondamental pour cette population, qui a politiquement et idéologiquement dominé le pays pendant si longtemps. Au cours des négociations entre l'ANC et le Parti national (NP), ces derniers ont constamment cherché à protéger la minorité afrikaner et à lui garantir un certain nombre de droits - d'aucuns diraient de privilèges. Puisque la domination politique n'était plus possible ni acceptable, le NP a tenté de masquer son but dans un discours sur la préservation des droits des groupes, de leur culture et de leur langue. Comme il est classique dans les démarches identitaires, il opposait là la communauté à l'individu et à la nation. Le groupe tel qu'il était vu est homogène et fermé sur l'extérieur. Il doit avoir ses propres écoles et universités, dans lesquelles on ne parle que sa propre langue. Cette vision est aussi celle d'intellectuels 
afrikaners particulièrement influents (Giliomee, 1996). La complexité des comportements individuels, le bilinguisme d'une grande partie de la population afrikaner, le fait que l'identité est quelque chose de mouvant, redéfini par chacun à chaque instant, ne sont absolument pas pris en compte. En bref, la défense officielle de l'identité afrikaner se fait de manière rigide, en concevant les groupes et les identités d'une manière similaire à celle pratiquée sous l'apartheid. Seule différence : puisque le critère le plus acceptable est celui de la culture et de la langue, on intègre dans ce groupe afrikaner un certain nombre de métis (Coloureds) dont l'afrikaans est la première langue.

Pour Behr et encore plus pour Antjie Krog, une redéfinition de l'identité afrikaner passe par la construction d'une nation sud-africaine. Avant toute chose, les Afrikaners doivent faire partie de cet ensemble plus large. L'un comme l'autre ont écrit en anglais, la lingua franca du pays. Pour parler de l'identité afrikaner et contribuer à la redéfinir, il faut donc s'adresser à l'ensemble de la nation et agir en accord avec elle. Le passé doit être dévoilé, accepté et assumé. Pour cela encore, le principal instrument utilisé est le langage. Le roman de Behr représente là une première étape : rappeler aux Sud-Africains du milieu des années 1990 la propagande gouvernementale des années d'apartheid, démontrer son horreur et son absurdité. Krog va plus loin. Tout d'abord, son lien avec la Commission Vérité et Réconciliation l'intègre dans un champ rhétorique particulier : sous l'influence entre autres de son président Desmond Tutu, la Commission a adopté un langage d'ordre religieux, évoquant le péché, la culpabilité, la confession et la rédemption (Aji et HoussayHolzschuch, 1997). Ensuite, le choix de la forme journalistique est motivé par la volonté suivante : en donnant la parole directement aux victimes, en reproduisant leur témoignage, elle s'oppose au langage de l'autorité repris par Marnus. Elle cherche à renverser l'autorité du discours en remplaçant celui, totalitaire et mensonger, du pouvoir, par ceux, multiples, véridiques et douloureux à l'extrême, des gens ordinaires qui ont été victimes. Elle pose une femme noire comme l'archétype de la victime, face à l'homme blanc, le bourreau, et donne la parole à la première : c'est elle qui détient la vérité et qui décide d'une éventuelle réconciliation. Dans son œuvre, la prise de parole reflète la prise de pouvoir, le langage est le lieu où s'instaure la démocratie (Krog, op. cit., p. 27 sq.). Les victimes sont noires comme blanches, elles parlent zoulou, anglais ou afrikaans. Les témoignages devant la Commission sont réellement, selon son expression, "la naissance du langage de ce pays ", enfin unifié.

Puisque pour Krog le langage est l'incarnation de la démocratie, l'utilisation de l'anglais était la seule possibilité : l'afrikaans est la langue de l'oppresseur, comme les Noirs l'ont proclamé depuis les émeutes de Soweto en 1976.

Le passé a donc été dévoilé dans toutes ses facettes, de celle révélée par Marnus à celles dévoilées par les multiples victimes. Le langage n'est plus instrument d'oppression, mais de libération et la nation naît dans la souffrance similaire ressentie par toutes les victimes. La dernière étape nécessaire à la redéfinition de l'identité afrikaner est d'accepter et d'assumer les horreurs du passé. Cela devrait être le rôle de l'élite politique afrikaner, rôle qu'aux yeux de Krog, de Klerk et ses ministres refusent d'assumer. En conséquence, les Blancs adoptent deux attitudes devant la Commission Vérité et Réconciliation : soit ils sont touchés et concernés mais se sentent impuissants devant l'énormité de la faute, soit ils refusent toute responsabilité personnelle et peuvent aller jusqu'à nier les faits eux-mêmes. Pour sortir de cette impasse, Krog fait appel à la distinction anthropologique classique entre culture de la honte (shame), collective et liée à une éthique de l'honneur, et culture de la culpabilité (guilt). Selon elle, la vie politique sud-africaine des années d'apartheid, qu'elle soit gouvernementale ou révolutionnaire, a pris place dans une culture de la honte. La référence était le groupe : on agissait en son nom, on défendait son honneur, on l'accroissait par ses actions. Les Afrikaners, comme certains leaders noirs à l'image de Winnie Mandela, sont encore dans cette éthique (Krog, op. cit., p. 262-263). La construction nationale ne peut se faire que si l'Afrique du Sud passe à une culture de la culpabilité, dans laquelle chaque individu est responsable de ce qu'il a commis. La Commission est l'instrument privilégié de cette transformation et l'adoption d'un langage religieux une nécessité logique.

Ainsi, la redéfinition d'une identité afrikaner dans la nouvelle Afrique du Sud ne pourrait se faire que dans une dialectique entre la nation et l'individu, puisqu'elle ne peut se faire, selon Krog, que par un cheminement individuel au sein d'un ensemble plus large, 
multiculturel et auquel on adhère volontairement : l'identité de chaque Afrikaner est désormais un choix.

\section{Conclusion}

Mark Behr et Antjie Krog participent tous les deux à la redéfinition de l'identité afrikaner dans la nouvelle Afrique du Sud. A travers une relecture du passé et l'appréhension du mal qui y a été commis, ils montrent comment chaque individu est aujourd'hui libre de déterminer son identité. En ce sens, leurs deux ouvrages sont deux réponses individuelles à la question : "comment être afrikaner aujourd'hui ?". Ils montrent les difficultés et les douleurs de cette quête, indiquent des chemins possibles.

Ils sont en même temps conscients des différenciations croissantes au sein de cette population. Quand ces différenciations sont le fait de choix individuels et ne visent pas simplement à perpétuer la domination raciale, ils ne peuvent que s'en féliciter. Mais la coupure entre la population et son élite est autrement plus grave, qu'il s'agisse de l'étatmajor "lâchant" les soldats se battant sur le front angolais ou de de Klerk n'aidant pas à assumer leur passé politique. Cette scission a été consommée par les négociations (Adam, Slabbert et Moodley, 1997) : l'élite afrikaner politique et socio-économique a conduit les négociations et s'est dessaisie du pouvoir, mais les catégories les plus défavorisées au sein de la population afrikaner n'adhéraient pas aux réformes. Elles ne pouvaient cependant s'exprimer, n'en ayant pas les moyens politiques - ceux-là même que les premiers cédaient. Ces catégories s'appauvrissent rapidement, n'étant plus protégées par la politique gouvernementale. Pourtant, la réconciliation nationale dépend en grande partie de leur attitude.

Le problème afrikaner reste central dans l'évolution de l'Afrique du Sud d'aujourd'hui. La réussite politique, comme la réussite économique, en dépendent largement. Plus encore, à cause d'un passé de privilégiés, les Afrikaners posent directement un certain nombre de question cruciales à la jeune démocratie : quelle place accorder au droit des groupes? Comment concevoir celui-ci autrement que comme la défense des privilèges, dans une conception exclusive ? Comment résoudre le dilemme entre état unitaire et multiculturalisme?

\section{Indications bibliographiques}

BEHR, M., 1993. Die Reuk van Appels. Strand, Queillerie.

BEHR, M. 1995. The Smell of Apples, London, Abacus, 200 p.

KROG, A., 1998. Country of my Skull, Johannesburg, Random House, 286 p.

ADAM, H. ; SLABBERT, F. van ZYL et MOODLEY, K. 1997. Comrades in Business: Post-Liberation Politics in South Africa. Cape Town, Tafelberg, 239 p.

AJI, H. et HOUSSAY-HOLZSCHUCH, M. 1997. “La Rhétorique de la réconciliation dans la nouvelle Afrique du Sud ", Esprit, ni 5 (mai), p. 90-107.

ASMAL, K. ; ASMAL, L. et ROBERTS, R. S. 1997. Reconciliation through Truth, A Reckoning of Apartheid's Criminal Governance, Cape Town, David Philip, 226 p.

GILIOMEE, H. 1996. “ Being Afrikaans in the New (Multilingual) South Africa”, New Contree, $\mathrm{n}_{i}$ 40, novembre, pp. 59-74.

HOUSSAY-HOLZSCHUCH, M. 1996. Mythologies territoriales en Afrique du Sud, un essai de géographie culturelle, Paris, CNRS, coll. Espaces et Milieux, 104 p.

HOUSSAY-HOLZSCHUCH, M. 1999. “L'Afrique du Sud ou la patrie utopique ”, dans Bonnemaison, J. ; Cambrézy, L. et Quinty-Bourgeois, L. 1999. Le Territoire, lien ou frontière ?, t. 2, Les Territoires de la nation. Paris, L'Harmattan, coll. Géographie et Cultures, p. 83-101. 
RESUME. Dans la nouvelle Afrique du Sud, les Afrikaners ont cédé le pouvoir politique. Leur identité, telle qu'elle s'était exprimée du temps de l'apartheid, reposait sur une vision exclusive du groupe, base de la ségrégation raciale. Elle doit aujourd'hui être redéfinie. Les intellectuels et les artistes participent à ce processus. L'analyse de deux ouvrages récents, The Smell of Apples de Mark Behr et Country of my Skull d'Antjie Krog, montre quelles sont les étapes nécessaires dans cette quête d'une nouvelle identité : reconnaître le mal commis sous l'apartheid, admettre qu'il est lié à une culture, l'assumer, pour pouvoir prendre place comme individu dans la jeune démocratie.

MOTS-CLEFS. Behr (Mark), Krog (Antjie), Afrikaner, identité, mémoire, Commission Vérité et Réconciliation, littérature, nouvelle Afrique du Sud.

ABSTRACT. In the New South Africa, the Afrikaners have negotiated themselves out of power. Their identity, as expressed during the apartheid regime, was based upon an exclusive vision of the group, leading to racial segregation. It must be redefined today, and intellectuals as well as artists are working on it. The analysis of two recent books, Mark Behr's The Smell of Apples and Antjie Krog's Country of my Skull, shows the steps that need to be taken and different possibilities in this quest for a new identity: to acknowledge the evil deeds that have been committed under apartheid, to admit they were linked to a specific culture, to assume them in order to take part, as an individual, in the young democracy.

KEYWORDS. Behr (Mark), Krog (Antjie), Afrikaner, identity, memory, Truth and Reconciliation Commission, literature, new South Africa. 